\title{
A Highly-verified biometric recognition system using an ultra-speed specifically- developed finger vein sensor
}

\author{
Khamis A. Zidan ${ }^{1}$, Shereen S. Jumaa ${ }^{2}$ \\ ${ }^{1}$ Al-Iraqia University, Iraq \\ ${ }^{2}$ Al-Nahrain University, College of Information Engineering, Iraq
}

\section{Article Info}

Received, 2019

\section{Keyword:}

Finger vein sensor

Methodology

ROI

Feature extraction.

\begin{abstract}
Currently, Biometrics has been utilized the top five modality of face, voice, IRIs, fingerprint, and palm to identify individuals. Comparatively, these Biometrics systems need complex computation to be slow and an easy target to hack. Alternatively, this work proposes a novel biometrics system of highly secured recognition with low computation time using specifically designed biometrics sensor. Consequently, finger vein recognition has been developed. Although, this recognition requires high point of safety measures comes with its individual experiments. The most prominent one being the vein pattern is very difficult to extract because finger vein images are constantly low in quality, seriously hampering the feature extraction and classification stages. Sophisticated algorithms need to be designed with the conventional hardware for capturing finger-vein images is modified by using red Surface Mounted Diode (SMD) leds. For capturing images, Canon 750D camera is used with micro lens. The integrated micro lens gives better quality images, and with some adjustments it can also capture finger print. Results have been comparatively improvement for SDUMLA-HMT database and extensively evaluated with k-nearest neighbors (KNN) algorithm. The (KNN) algorithm is a simple, easy-to-implement supervised machine learning algorithm that can be used to solve both classification and regression problems. KNN calculations are highly accurate in test data. Using stratified 6- fold analysis on all fingers of all hands in collected database, a maximum accuracy of $100 \%$ was achieved with an EER of $0 \%$ when select right hand and middle finger, based on the analysis of the 106 persons present in the data set. Many approaches have been used to optimize vein image quality. The proposed system has optimum results as compared to existing related works. The work novelty is due to the hardware design of the sensor within the finger-vein recognition system to obtain, simultaneously, finger vein and finger print at low cost, unlimited users for one device and open source.
\end{abstract}

\section{Corresponding Author:}

First Author,

Al-Iraqia University, Iraq

Email: khamis_zidan@aliraqia.edu.iq

\section{Introduction}

Bio-metric means measurement of body anatomic characteristics. These characteristics are either extrinsic or intrinsic. Extrinsic characteristic includes fingerprint, retina, iris scans, facial and hand patterns, gait, voice analysis, signature and intrinsic characteristic are finger-vein patterns, DNA.

These characteristics vary from person to person [1] [2] [3].

Bio-metric measures are also used for personal security authentication. In fingerprint scan, fingerprint impression of a person is recorded optically and stored which is later used for identity verification. Retinal scan records patterns of blood vessels of retina. In iris scanning pattern of iris is captured and used for verification. In face verification system, facial features are captured for verification. Deoxyribonucleic acid 
(DNA) is hereditary material in humans. It is a helix structure found in every cell and almost every cell has same DNA composition, and unique from person to person. It is also used for person verification process. In finger-vein verification method, finger-vein pattern is captured and used for verification [4] [5] [6] [ 7]. In finger vein recognition method, images of the finger vein patterns are captured and then matched with the previously obtained data. The patterns of finger vein are captured by exposing finger to near infrared (NIR) light emitting diode light. The hemoglobin in the blood vessels absorbs this light and a camera records the image. The near infrared light which is absorbed appears black in image while the rest of the finger appears white. The image is then digitized for processing. The distance between skin and veins is very important. If the distance is large, the image created will be very noisy, and recognition of the pattern will be very hard[8] [9] [10].Bio-metric systems based on vein recognition are not only for fingers, but are also used to recognize vascular properties of retina, face and hands. This method has been successful in receiving the attention of masses due to its high authentication accuracy and as the pattern is invisible, which makes it very hard to be spoofed. The typical framework of the biometric finger vein recognition (FVR) system is presented in(see figure 1).

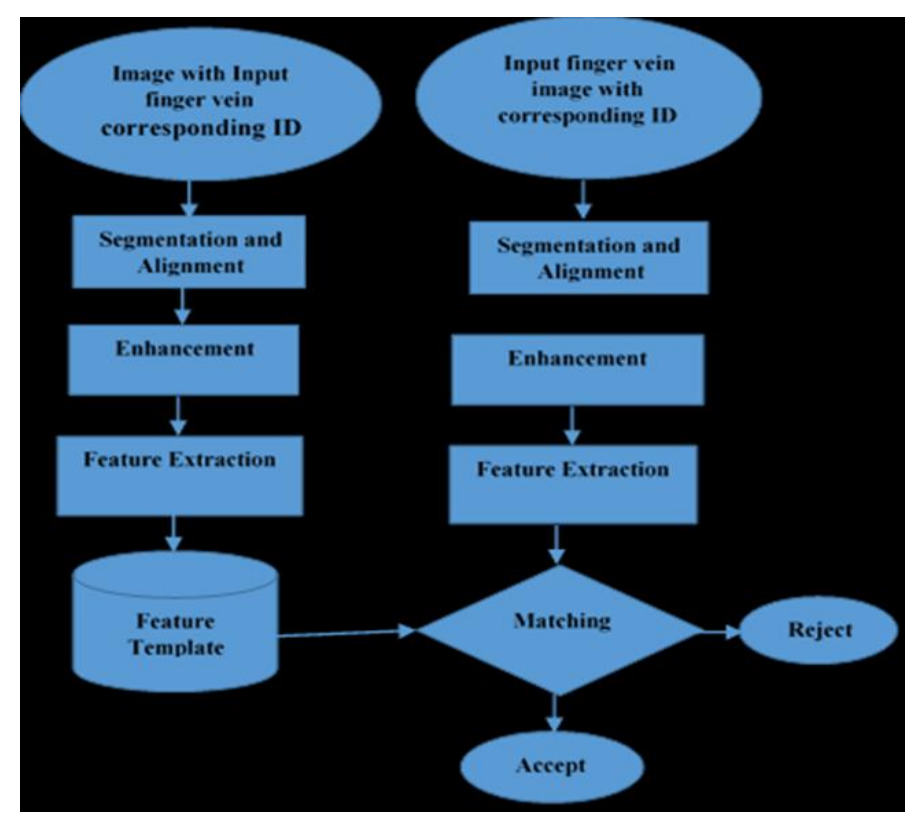

Figure 1. Finger-vein recognition process [11]

\section{Database}

The main problem in finger-vein recognition is to remove noise from the acquired images, and to do recognition in shortest amount of time. This can be achieved by optimizing the hardware to acquire good quality images and by optimizing the image processing techniques [6] [7].

In this paper finger-vein recognition system is optimized by developing a novel way for image capturing in the hardware of the device, and the acquired images are processed by enhancing the finger-vein recognition stages, that evaluates a set of enhancement stages for finger vein enhancement that not only has low computational complexity but also high distinguishing power.

The amount of light that will be delivered by infrared led will vary according to thickness of finger.The main problem in finger-vein recognition is to remove noise from the acquired images, and to do recognition in shortest amount of time. This can be achieved by optimizing the hardware to acquire good quality images and by optimizing the image processing techniques.

In this research finger-vein recognition system is optimized by developing a novel way for image capturing in the hardware of the device, and the acquired images are processed by enhancing the finger-vein recognition stages, that evaluates a set of enhancement stages for finger vein enhancement that not only has low computational complexity but also high distinguishing power.

To evaluate our algorithm, we collected database by proposed device. A number of people who take part is one hundred (100). The format of images of vein is .jpg. Of each person, images of both two hands were taken. Data of three fingers were collected i.e. index, middle and ring fingers for each hand and for each finger, 6 samples were obtained as shown in(see figure 2 and table 1). 


\begin{tabular}{|l|l|}
\hline Parameter & Table 1. Database of a finger vein \\
\hline Total persons & 100 \\
\hline Hands per person & 2 \\
\hline Fingers per hand & 3 \\
\hline Images per finger & 6 \\
\hline Image resolution & $910 \times 603$ \\
\hline Image format & JPG \\
\hline
\end{tabular}

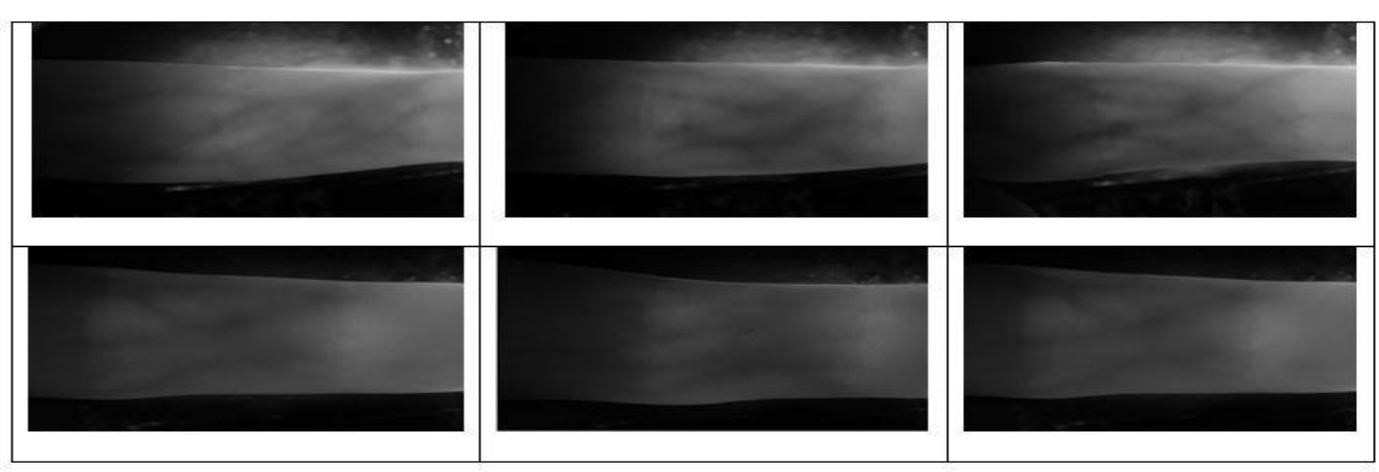

Figure 2. Database-sample data

\section{Finger vein sensor}

In this research the conventional hardware for capturing finger-vein images is modified by using red Surface Mounted Diode (SMD) leds on both sides, instead of near-infrared leds. For capturing images, Canon 750D camera is used with micro lens. The integrated micro lens gives better quality images, and with some adjustments it can also capture finger print. The canon camera used for image capturing can be replaced by mobile camera. The SMD leds that are used have better illumination. The objective of this paper is to increase accuracy and efficiency of finger-vein recognition by reducing the processing time. To achieve this goal, the hardware of finger-vein recognition device is modified by using SMD leds instead of near-infrared leds, and a micro lens is integrated with the camera to improve images quality by generating high quality images. Finger vein sensor as shown in (see figure 3).

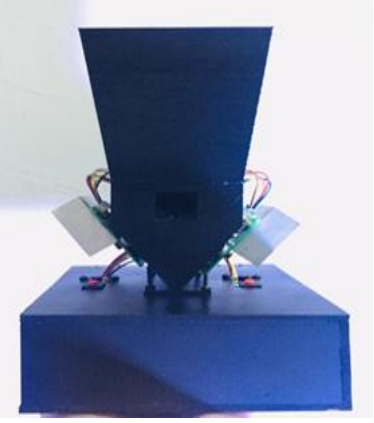

(a)

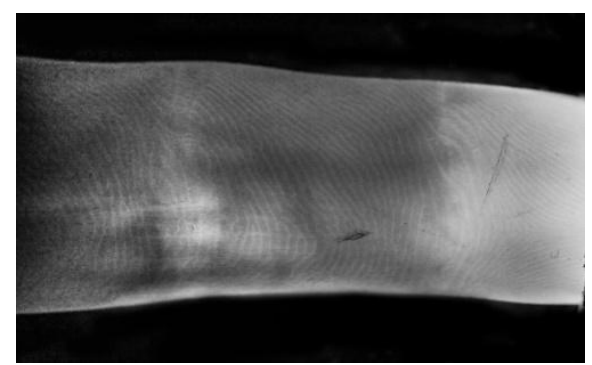

(b)

Figure 3. (a) Image capturing device (b) Sample of finger-vein image

\section{Methodlogy}

Finger-vein recognition is a interesting job because it gives the low quality of images due to distorted vein patterns and vein substances and irregular illumination which reduces the efficiency of the Finger Vein recognition method. In this way, it is important to increase the nature of finger vein images for finger vein recognition system. To achieve effective recognition performance, the image acquired is preprocessed in different stages. The algorithm is evaluated as following : 
4.1 The region of interest (ROI) of the finger vein image must be segmented and the nature of finger vein image enhancement must be assessed. To do this localization, the filter that is used is mask filter which follows the procedure as [12] [13]. The database image is then cropped by keeping the portion of only finger region as shown in(see figure 4 )

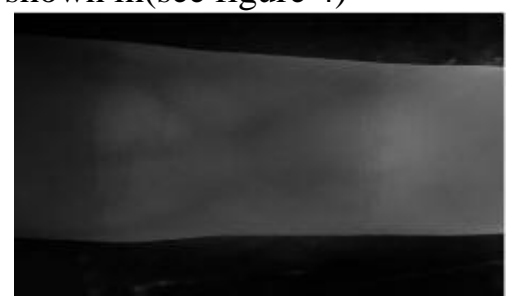

(a)

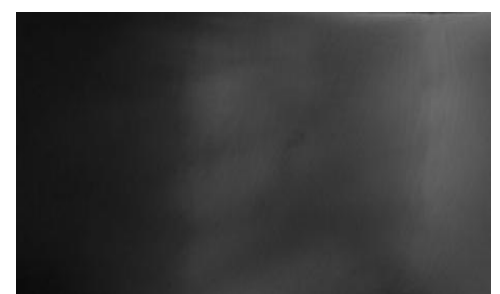

(b)

Figure 4. (a) Database image, (b) Cropped finger

\subsection{Gamma correction}

Using Gamma correction, brightness of the cropped finger is enhanced [14] [15]. Manipulating the pixels nonlinearly, enhances the dynamic range of pixel intensities. It is obtained by minimizing the homogeneity of cooccurrence matrix [16] [17]. It raises the quality of image effectively by steering the average brightness of an image in the direction desired. In proposed algorithm, it was observed that if gamma parameter is kept to be 2 , it gives most optimum results as shown in the image in (see figure 5).

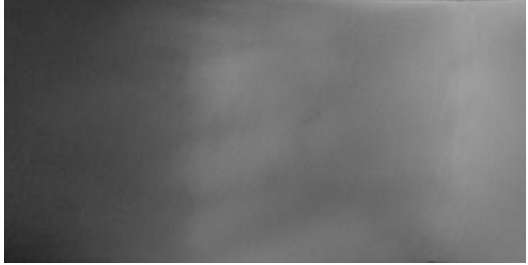

(a)

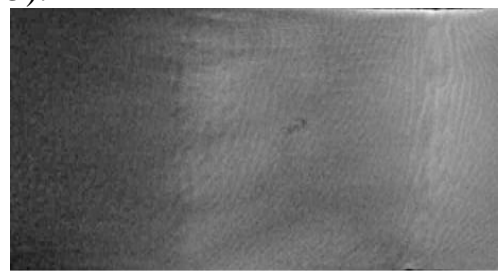

(b)

Figure 5. (a) An image after gamma correction, (b) An image after sharpening

\subsection{Fuzzy histogram equalization}

After getting high quality images, fuzzy histogram equalization is used to enhance veins illumination stages. Dynamic fuzzy histogram equalization does not affect the brightness [18]and it gives the histogram image in a way that in mapping of peaks does not arise again. The values of histogram are spread again in a uniform way between valley similar areas of two serial peaks [18].In the figure 6 The image after fuzzy histogram equalization and image histogram.

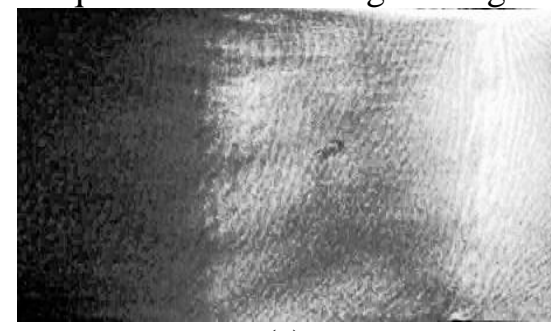

(a)

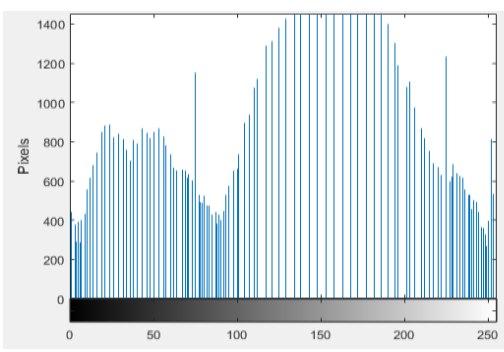

(b)

\subsection{Median filtering}

Figure 6. (a) An image after histogram equalization, (b) An image histogram

Median filtering is a non-linear process. To remove the impulsive and salt-and-pepper noise, median filtering is done on the image. It smoothly removes noise and preserves the edge-information. Strength of median filter depends upon window size [19] [20] [21](see in figure 7).

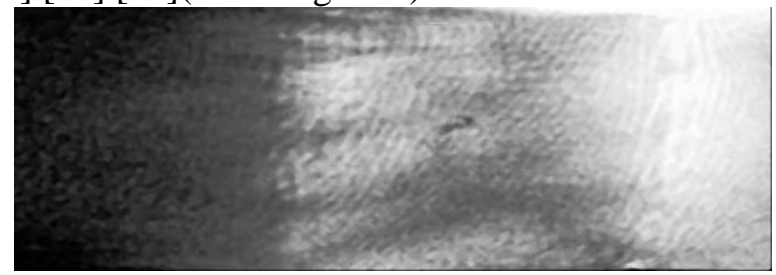

Figure 7. An image after median filter enhancement 


\section{Feature extraction}

For feature extraction from images, histogram of oriented gradient (HOG) and hierarchical centroid shape descriptor methods are used. Both enhancement stages are evaluated with K Nearest Neighbor and Deep Neural Networks using 6 fold stratified cross validation[22] [23] [24] [25] [26].

The proposed solution framework for solving the challenges of the finger vein pattern using k-mean classification technique is proposed. The proposed algorithm evaluates the quality of the enhanced vein image, which produces improved vein features. The proposed approach efficiently classifies the finger vein pattern based on the extracted features, and improves finger vein recognition system [27] [28] [29] [30] [31] [32].

\section{Results}

The computational time for each phase of the proposed enhancement methods is shown in (see table 2) below.

Table 2. Average computational time per step for proposed method

\begin{tabular}{|l|l|}
\hline Preprocessing phases of proposed method & $\begin{array}{l}\text { Average time taken } \\
\text { (seconds) }\end{array}$ \\
\hline finger detection & 0.0072 \\
\hline gamma correction & 0.0011 \\
\hline image sharpening & 0.0036 \\
\hline fuzzy histogram equalization & 0.0041 \\
\hline median filtering & 0.0059 \\
\hline total time & 0.0219 \\
\hline
\end{tabular}

An extensive evaluation of the algorithm was implemented. The mix finger type and hand type was tested for each feature extraction method and also for each classifier. In the experiment, it was found that the middle finger is the best of all for the considered database of 106 persons. For the experimental results shown below, each finger of each hand is taken a distinct set as in (see figure 8), even if it is of the same person.

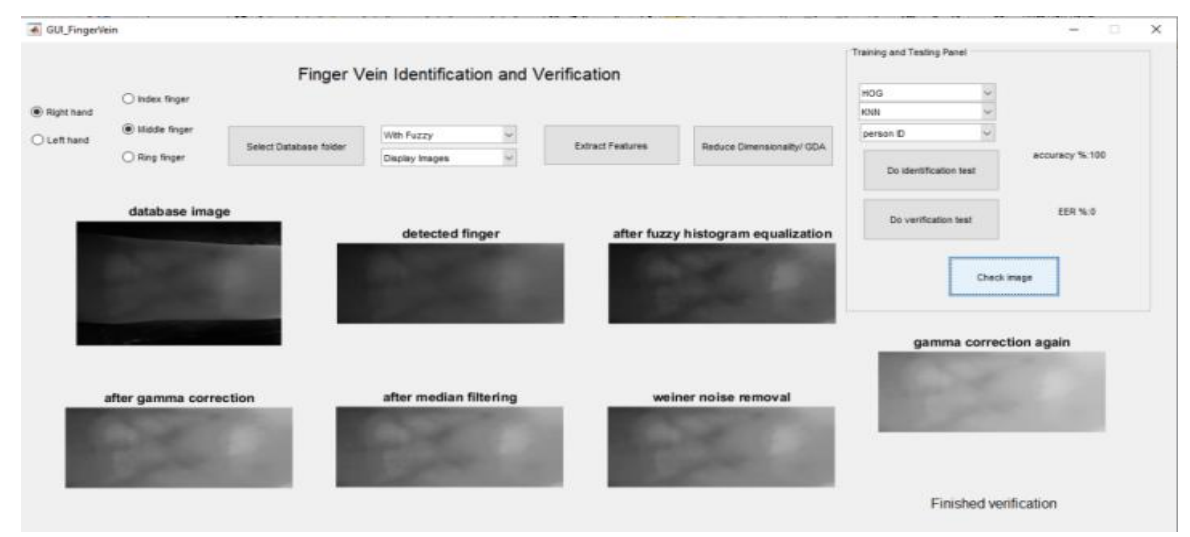

Figure 8. GUI for finger recognition and verification

Results of the proposed algorithm for recognition and verification for middle finger of right hand, left hand and both hands are collected in the tables below. For KNN, results are given with HOG, HC and with the feature vector of HOG and HC. For DNN, dimensions of combined HOG and HC were first reduced (to be equal to 15) using Generalized Discriminant Analysis (GDA) [33], before giving them for classification. The cause for this is that data samples per class were limited and DNN must work best on wide-ranging of data whose feature length is not more than the total available samples.

Table 3 . Results of KNN with right hand, middle finger

\begin{tabular}{|l|l|l|}
\hline Method & $\begin{array}{l}\text { Accuracy } \\
(\mathbf{\%})\end{array}$ & EER (\%) \\
\hline Proposed approach using HOG & 100 & 0 \\
\hline Proposed approach using HC & 95.2381 & 0.22676 \\
\hline Proposed approach using HOG+HC & 100 & 0 \\
\hline
\end{tabular}


Table 4. Results of KNN with left hand, middle finger

\begin{tabular}{|l|l|l|}
\hline Method & Accuracy (\%) & EER (\%) \\
\hline Proposed approach using HOG & 80.9524 & 0.90703 \\
\hline Proposed approach using HC & 90.4762 & 0.45351 \\
\hline Proposed approach using HOG+HC & 80.9524 & 0.90703 \\
\hline
\end{tabular}

Table 5. Results of KNN with both Right and Left Hand, Middle Finger

\begin{tabular}{|l|l|l|}
\hline Method & Accuracy (\%) & EER (\%) \\
\hline proposed approach using HOG & 90.4762 & 0.45351 \\
\hline proposed approach using HC & 92.8571 & 0.34014 \\
\hline proposed approach using HOG+HC & 90.4762 & 0.45351 \\
\hline
\end{tabular}

From the above results, it can be decided that KNN gives a better results with right hand with middle finger Reason that have more vein with extensive data. We also achieved analysis of the proposed way using all fingers of both hands (636 set classification) present in the database for comparison with some state of the art recent benchmarks in this field(see table 6).

Table 6. Results with all fingers of both hands with stratified 6 fold cross validation

\begin{tabular}{|c|c|c|c|}
\hline Reference & Method & $\begin{array}{l}\text { Accuracy } \\
(\%)\end{array}$ & $\operatorname{EER}(\%)$ \\
\hline [34] & $\begin{array}{l}\text { Tri-Branch Vein } \\
\text { Structure } \\
\text { Analysis }\end{array}$ & - & 3.46 \\
\hline [10] & $\begin{array}{l}\text { Anatomy } \\
\text { Structure } \\
\text { Analysis }\end{array}$ & - & 1.39 \\
\hline [8] & $\begin{array}{l}\text { Deformation } \\
\text { information }\end{array}$ & 94 & 0.0268 \\
\hline [35] & $\begin{array}{l}\text { Proposed } \\
\text { approach using } \\
\text { CHFHE with } \\
\text { HOG+HC+KNN }\end{array}$ & 95.8857 & 0.0123 \\
\hline \multirow[t]{2}{*}{ [35] } & $\begin{array}{l}\text { Proposed } \\
\text { approach using } \\
\text { FHE with } \\
\text { HOG+HC+KNN }\end{array}$ & 97.6415 & 0.0037 \\
\hline & $\begin{array}{l}\text { Proposed } \\
\text { approach using } \\
\text { HOG+HG+KNN } \\
\text { with proposed } \\
\text { sensor }\end{array}$ & 100 & $\mathbf{0}$ \\
\hline
\end{tabular}

\section{Conclusion and future work}

A novel method has been developed of finger vein enhancement for recognition and verification purposes. The results obtained from this research to increase accuracy and reduce processing time for finger-vein recognition will add a significant contribution in the field of biometrics recognition systems. The approach developed in this research has optimum results as compared to previous ones.

For future work, The developed system may be optimized using some optimization methods [36], furthermore, using features vein and finger print in classification and recognition approach. Convolution Neural Networks [37] may efficiently be used to check performance using transfer learning techniques.

\section{References}

[1] K. Shaheed, H. Liu, G. Yang, I. Qureshi, J. Gou and Y. Yin, "A Systematic Review of Finger Vein Recognition Techniques," MDPI, 2018.

[2] A. R. A. A. N. K. Jain, "Introduction to Biometrics", Springer, 2011. 
[3] K. N. Mishra, N. C. Mishra and A. Agrawal, "Veins Based Personal Identification Systems: A Review," I.J. Intelligent Systems and Applications,, 2016.

[4] A. Kumar and Y. Zhou, "Human Identification Using Finger Images," IEEE Transactions on Image Processing, 2011.

[5] W. Xuebing, Z. Jiangwei and L. Xuezhang, "Research on enhancing human finger vein pattern characteristics," Asia-Pacific Conference on Power Electronics and Design, 2010.

[6] E. C. Lee and K. R. Park, "Image restoration of skin scattering and optical blurring for finger vein recognition," Optics and Lasers in Engineering, 2011.

[7] L. Yang, G. Yang, Y. Yin and X. Xi, "Finger Vein Recognition With Anatomy Structure Analysis," IEEE Transactions on Circuits and Systems for Video Technology, 2018.

[8] X. M. X. Y. Yin, Science China Information Sciences," Finger vein recognition based on deformation information", 2018.

[9] Y. Yin and L. L. X. Sun, "SDUMLA-HMT: A Multimodal Biometric Database," in Chinese Conference on Biometric Recognition, 2011.

[10] G. Y. Y. Y. a. X. X. Lu Yang, "Finger Vein Recognition with Anatomy Structure Analysis," IEEE TRANSACTIONS ON CIRCUITS AND SYSTEMS FOR VIDEO TECHNOLOGY, 2017.

[11] X. Xi, L. Yang and Y. Yin, "Learning discriminative binary codes for finger vein recognition," Pattern Recognition, 2017.

[12] K. Akintoye, M. S. M. Rahim and A. H. Abdullah, "ENHANCEMENT OF FINGER VEIN IMAGE USING MULTIFILTERING ALGORITHM," ARPN Journal of Engineering and Applied Sciences, 2018

[13] H. C. L. K. R. P. Eui Chul Lee, "Finger Vein Recognition Using Minutia-Based Alignment and Local Binary Pattern-Based Feature Extraction".

[14] S. A. Amiri and H. Hassanpour, "A Preprocessing Approach For Image Analysis Using Gamma Correction," International Journal of Computer Applications , 2012.

[15] K. Somasundaram and P. Kalavathi, "MEDICAL IMAGE CONTRAST ENHANCEMENT BASED ON GAMMA CORRECTION," International Journal of Knowledge Management \& e-Learning, 2011.

[16] C. W. ,. S. L. ,. M. R. ,. T. R. ,. N. K. ,. H. S. ,. Y.-H. Y. T. W. G. Jianga, "Image contrast enhancement with brightness preservation using an optimal gamma correction and weighted sum approach," Journal of Modern Optics, 2015.

[17] C. W. ,. S. L. ,. M. R. ,. T. R. ,. N. K. ,. H. S. ,. Y.-H. Y. T. W. G. Jianga, "Image contrast enhancement with brightness preservation using an optimal gamma correction and weighted sum approach," Journal of Modern Optics, 2015.

[18] D. Sheet, H. Garud, A. Suveer, M. Mahadevappa and J. Chatterjee, "Brightness Preserving Dynamic Fuzzy Histogram Equalization," IEEE Transactions on Consumer Electronics, 2010.

[19] H. Hassanpour, N. Samadiani and S. Salehi, "Using morphological transforms to enhance the contrast of medical images," The Egyptian Journal of Radiology and Nuclear Medicine, 2015.

[20] M. Nagu and N. Shanker, "Image De-Noising By Using Median Filter and Weiner Filter," International Journal of Innovative Research in Computer and Communication Engineering, 2014.

[21] A. Makandar and B. Halalli, "Breast Cancer Image Enhancement using Median Filter and CLAHE," International Journal of Scientific \& Engineering Research, 2015.

[22] S. A. Korkmaz, A. Akçiçek, H. Bínol and M. F. Korkmaz, "Recognition of the stomach cancer images with probabilistic HOG feature vector histograms by using HOG features," in 2017 IEEE 15th International Symposium on Intelligent Systems and Informatics (SISY), 2017.

[23] J. Ouyang, "Combining Extreme Learning Machine, RF and HOG for Feature Extraction," in IEEE Third International Conference on Multimedia Big Data (BigMM), 2017.

[24] S. A. Korkmaz, A. Akçiçek, H. Bínol and M. F. Korkmaz, "Recognition of the stomach cancer images with probabilistic HOG feature vector histograms by using HOG features," in IEEE 15th International Symposium on Intelligent Systems and Informatics (SISY), 2017.

[25] J. Chen, D. Zhou, Y. Wang, H. Fu and M. Wang, "Image feature extraction based on HOG and its application to fault diagnosis for rotating machinery," Journal of Intelligent \& Fuzzy Systems, 2018.

[26] C. Q. Lai and S. S. Teoh, "Efficiency Improvement in the Extraction of Histogram Oriented Gradient Feature for Human Detection Using Selective Histogram Bins and PCA," in 9th International Conference on Robotic, Vision, Signal Processing and Power Applications, 2016. 
[27] E. Ilunga-Mbuyamba, J. G. Avina-Cervantes, D. Lindner and J. Guerrero-Turrubiate, "Automatic brain tumor tissue detection based on hierarchical centroid shape descriptor in Tl-weighted MR images," in 2016 International Conference on Electronics, Communications and Computers (CONIELECOMP), 2016.

[28] L. K. J. H. K.-H. J. Wahyono, "Similarity-based classification of 2-d shape using centroid-based tree-structured descriptor," Modern Advances in Applied Intelligence, Springer International Publishing, 2014.

[29] S. Zhang, X. Li, M. Zong, X. Zhu and R. Wang, "Efficient kNN Classification With Different Numbers of Nearest Neighbors," IEEE Transactions on Neural Networks and Learning Systems , 2018

[30] A. Z. A. Zainuddin, W. Mansor, L. Y. Khuan and Z. Mahmoodin, "Classification of EEG Signal from Capable Dyslexic and Normal Children Using KNN," American Scientific Publishers, 2018.

[31] M. S. Sarma, Y. Srinivas, M. Abhiram, L. Ullala and M. S. Prasanthi, "Insider Threat Detection with Face Recognition and KNN User Classification," in IEEE International Conference on Cloud Computing in Emerging Markets (CCEM), 2017.

[32] R. S. P. S.Ponmani, "Classification Algorithms in Data Mining - A Survey," International Journal of Advanced Research in Computer Engineering \& Technology (IJARCET), 2017.

[33] F. Bahmaninezhad and J. H. Hansen, "Generalized Discriminant Analysis (GDA) for Improved iVector Based Speaker Recognition," INTERSPEECH, 2016.

[34] L. Yang, G. Yang, X. Xi, X. Meng, C. Zhang and Y. Yin, "Tri-Branch Vein Structure Assisted Finger Vein Recognition," IEEE Access , vol. 5, 2017.

[35] K.Zidan and Sh.Jumaa ,"Finger vein recognition using two parallel enhancement approaches based fuzzy histogram equalization",PEN,vol.7,2019 .

[36] P. Sun, W. Feng, R. Han, S. Yan and Y. Wen, "Optimizing Network Performance for Distributed DNN Training on GPU Clusters: ImageNet/AlexNet Training in 1.5 Minutes," Distributed, Parallel, and Cluster Computing, 2019.

[37] M. Jamal, A. Arun, RossaErik and M.Shapirob, "On automated source selection for transfer learning in convolutional neural networks," Pattern recognition, 2018. 